\title{
Nordic research infrastructures for plant phenotyping
}

\author{
Erik Alexandersson ${ }^{1,2}$, Markku Keinänen ${ }^{3,4}$, Aakash Chawade ${ }^{5}$, Kristiina Himanen ${ }^{3,6}$ \\ ${ }^{1}$ Department of Plant Protection Biology, Swedish University of Agricultural Sciences, P.O. Box 102, SE-23053 Alnarp, Sweden- \\ ${ }^{2}$ PlantLink, www.plantlink.se \\ ${ }^{3}$ National Plant Phenotyping Infrastructure, Kanslerinkaari 2, 00790 Helsinki, Finland \\ ${ }^{4}$ Department of Environmental and Biological Sciences, University of Eastern Finland, PO Box 111, 80101 Joensuu, Finland \\ ${ }^{5}$ Department of Plant Breeding, Swedish University of Agricultural Sciences, Alnarp, Sweden \\ ${ }^{6}$ University of Helsinki, Department of Agricultural Sciences, Latokartanonkaari 7, 00790 Helsinki, Finland
}

e-mail: kristiina.himanen@helsinki.fi

\begin{abstract}
Plant phenomics refers to the systematic study of plant phenotypes. Together with closely monitored, controlled climates, it provides an essential component for the integrated analysis of genotype-phenotype-environment interactions. Currently, several plant growth and phenotyping facilities are under establishment globally, and numerous facilities are already in use. Alongside the development of the research infrastructures, several national and international networks have been established to support shared use of the new methodology. In this review, an overview is given of the Nordic plant phenotyping and climate control facilities. Since many areas of phenomics such as sensor-based phenotyping, image analysis and data standards are still developing, promotion of educational and networking activities is especially important. These facilities and networks will be instrumental in tackling plant breeding and plant protection challenges. They will also provide possibilities to study wild species and their ecological interactions under changing Nordic climate conditions.
\end{abstract}

Key words: plant phenomics, climate control, imaging sensors, growth facilities, networking

\section{The role of plant phenomics and controlled environments in response to climate change}

Climate change is affecting plants in all ecosystems and poses new challenges for agriculture and forestry. The changing climate is altering the European agricultural landscape by moving the most favorable areas towards the currently less favorable areas in the North, while creating challenges for sustainable crop cultivation in some Southern regions. The Nordic region, represented in this review by Fennoscandia, will face a set of specific climate challenges. Nordic agriculture currently lies at the border of the cultivation zone for most crops because the growth season is exceptionally short and mean temperatures are low (Peltonen-Sainio 2012). In the region, fluctuating weather conditions are already a great challenge for agriculture practices and as the climate is warming up, weather fluctuations are predicted to increase further. In the Nordic ecosystems, the warmer autumns and milder winters will also lead to new plant pathogens and plant invasive species migrating north leading to an increased pathogen pressure (Roos et al. 2011, van der Fels-Klerx et al. 2013). For example, in the summer of 2012, the mean vegeta-tion greenness index (NDVI) was recorded to be the lowest in the preceding 10 years in the Nordic Arctic region due to adverse weather events and insect and fungal disease (Bjerke et al. 2014). Such events might well be further intensified by the predicted increase in precipitation and air humidity at high latitudes (IPCC 2013). Furthermore, precipitation is projected to increase in Northern Europe throughout the year, whereas, elsewhere in Europe, the summers are predicted to get dryer (Peltonen-Sainio 2012). Increasing atmospheric humidity reduces transpira-tion water flux through plants, potentially diminishing nutrient uptake and leading to an unbalanced foliar phos-phorus/nitrogen ratio, resulting in decline in leaf photosynthetic capacity (Sellin et al. 2017). Increased air humid-ity has also been shown to affect leaf surface wax composition both in a controlled conditions and in the field, re-sulting to less hydrophobic wax composition in Silver birch leaves (Lihavainen et al. 2017). Changes in leaf surface properties are likely to affect leaf wettability, cuticular permeability and resistance to pathogens and herbivores (Deepak et al. 2018), but may also affect responses to foliar spray applications, such as herbicides. A change in the weed flora is also predicted (Andreasen and Streibig 2011). Thus, breeding for climate adaptation includes both tolerance to abiotic stresses (drought, frost, heat and flooding) and biotic stresses (pests and pathogens), and crop performance traits, such as water and nutrient use efficiency (WUE, NUE).

To allow fast responses in agriculture practices to the future climate challenges new plant breeding tech-nologies are urgently needed. Plant phenomics refers to high throughput assessment of plant trait re-sponses to genomic and environmental variation. Automation of plant phenotyping in association with controlled climate facilities will allow testing crop performance in predicted future climate scenarios. 
High-throughput non-invasive phenotyping can become a powerful tool for plant breeding, precisions agriculture and trait-based ecology where phenotypes of complex traits can be identified. Efficient screening of genetic resources for plant performance and stress resilience in simulated climate conditions will be key to secure access to sufficient, safe and nutritious food for the growing world population (Wheeler and von Braun 2013).

\section{Phenotyping in controlled environments for agriculture and plant ecology}

Advanced climate chambers offer multiple and precise climate conditions to allow dissection of the relationships between plant traits and specific environmental factors. Climate control that includes adjustable temperature and humidity conditions, application of atmospheric gas exchange and supplements, different schemes and ranges of nutrients, controlled water status and wavelength adjustable illumination, allows plant exposure to different stresses, testing different climate scenarios, and ultimately modeling crop performance. Controlled climate chambers are useful to study key concepts in agriculture, plant ecology and botany such as fitness and local adaptation. Large-scale phenotyping can be a powerful way to estimate the growth dynamics and fitness of large plant populations as recently demonstrated for Arabidopsis by imaging in either semi-automated or automated highthroughput modes (Pavicic et al. 2017, Vasseur et al. 2017).

A controlled environment can be used similarly to a common garden experiment where several genotypes can be tested in parallel for local adaptation and explore the relationships between phenotype, genotype and environment. In addition, controlled climates can be used to simulate local climates, as for example done in a study of 472 Arabidopsis accessions where climate chambers were used to mimic the growth season in Sweden and Spain, in order to map climate-sensitive QTLs related to flowering time (Li et al. 2010). Other phenomena difficult to test in outdoor conditions with varying climates such as epigenetic effects can also be efficiently studied in controlled environments. For example, in a study of the wild potato relative Solanum physalifolium, it was shown that an accession collected from Southern Sweden showed a non-genetic inheritance of induced resistance (Lankinen et al. 2016).

Trait-based ecology has emerged as an alternative to species compositions to describe ecosystems. Measurements of traits rather than species composition can be more powerful because traits are more closely connected to and influenced by the environment. Also in evolutionary biology it is an alternative to follow certain traits that species carry rather than the species themselves. Trait-based approaches requires linking accurate phenotypic analysis of traits with environmental conditions. Here, the common focus on single instead of multiple traits is a challenge that has been identified and needs to be addressed (Laughlin and Messier 2015). A better understanding and recording of multidimensional phenotypes, representing both growth and physiology, is a key in studying the indirect link between traits and community dynamics. Furthermore, stronger empirical links between functional traits and fitness components needs to be established. Controlled environments in combination with phenomics facilities is one way to scale up the number of phenotypes measured in a more standardized way. In addition, such set-ups can be used to identify variation within a species, rather than the current focus on variation between species (Shipley et al. 2016). We anticipate that plant ecologists and evolutionary biologists will have great use of the emerging Nordic facilities and networks around plant phenomics, and that these will help to bridge these disciplines to plant breeding and stress biology.

\section{Imaging sensors and phenomics data management}

Detecting and quantifying plant appearance is challenging and prone to subjective bias if done by visual inspection. Human color perception is affected by context (color contrast and assimilation effects) and illumination intensity. Furthermore, the human eye is not equally sensitive to all wavelengths of visible light, and subject to metamerism: a variety of wavelength band combinations may produce the same observed color. Currently, most plant phenotyping studies are based on RGB (Red Green Blue) imaging, although thermal and especially chlorophyll fluorescence imaging is also widely adopted (Humplík et al. 2015). Conventional RGB cameras provide quantifiable information but are also limited to three broad and partly overlapping bands of the sensor Bayer filter. For detection of subtle spectral differences, spectroscopic approaches must be used. Spectral variation in the plant reflects differences in structural properties, chemical composition and variation in physical attributes in local microenvironments, all of which may vary among genotypes or in response to environmental stress factors. Hyperspectral imaging (HSI) enables the detection of hundreds of wavebands, and can be applied in different spectral domains, from UV to infrared. Visible to near infrared (VNIR, 400-1000nm) and short-wave infrared (SWIR, 1000-2500nm), HSI systems are most common, and particularly well suited for detecting spectral changes through time, as in plant disease dynamics. Disease progression can thus be visualized as reflectance trajectories through time, each disease exhibiting a specific route with the direction and steps corresponding to biological processes (Wahabzada et al. 
2015). HSI systems at the infrared range can be used to reveal differences also in the inner structure of plant parts, such as in screening of spruce seeds for viability and pest infestation (Dumont et al. 2015). Thus, sensor-based phenotyping allows non-invasive analysis of both plant growth and physiology and thereby facilitates especially time course experiments. In combination with automated plant management, such as transportation, randomization and watering, the labor of phenotyping hundreds of plants simultaneously is significantly reduced allowing increased throughput (Pavicic et al. 2017).

This development has essentially created a critical mass of data to allow for the shared international efforts in standardizing also the data management. Plant phenotyping performed until now has not been systematic or standardized enough in experimental design or data collection. The data sets often also lack appropriate metadata, such as logging of the environmental records. Importantly, moving plant analysis to high-throughput setting opens the way for the future of "open data" also for phenomics. Good repositories have to be developed for these large-size and multi-faceted data sets to be stored in an organized way (Arend et al. 2016, Coppens et al. 2017). Today, $71 \%$ of all biological datasets stay on local hard drives, where they will never be re-used and are eventually lost within 10-15 years (Stanford et al. 2015). To avoid this, a change of mindset is required amongst biologists in combination with the assistance from researchers experienced in handling big data. The next steps in plant phenomics should, therefore, focus on developing standards for both data and metadata (Krajewski et al. 2015). Such standards are being formulated in the frame of Minimum Information About a Plant Phenotyping Experiment (MIAPPE, http://www.miappe.org/), Plant Breeding API (BRAPI, https://brapi.org/) and European research infrastructure networks, such as ESFRI (EMPHASIS) and ELIXIR (EXCELERATE), to tackle technical challenges in unifying data collection. In addition, serious attempts will be made to integrate phenomics data with molecular omics data and especially the genomics data that is already available in open databases.

\section{Survey of Nordic phenomics and climate control facilities}

To address the grand challenges related to climate change, sustainable food and feed production, and future crop performance in the Nordic countries, there is a need to further develop and increase the use of plant phenomics and controlled growth facilities. In this review, we therefore surveyed the existing facilities for Nordic phenomics and climate control used for plant research. We believe that an overview of available facilities will be valuable for Nordic plant researchers to be able to pursue the testing of future climate scenarios and their effects on plant ecosystems, forestry and crop production. We also suggest a number of actions that scientists in the Nordic region could take to advance the research on plants in future climates and which can provide more efficient schemes for plant breeding and protection.

There has been a boom in newly established plant phenomics facilities over the last few years (for a comprehensive worldwide list of facilities see www.plant-phenotyping.org), and in addition many labs are developing their own systems. To obtain an overview of current state of the climate control chambers and high-throughput phenotyping facilities available in the Nordic countries, we sent out a survey to 13 Nordic academic institutions. The survey results are summarized in Table 1.

The survey shows that several new facilities for plant phenotyping and growth have been established in the Nordic countries since 2008 and the universities in Aarhus, Copenhagen, Helsinki and Stockholm as well as SLU in Alnarp have inaugurated new facilities in 2015-2016. Others have up-graded their facilities, especially with LED lighting and more advanced cameras and sensors for phenotyping. There is also a recent trend with adaptions to larger plants such as crops and trees in custom-made facilities, whereas, off-the-shelf cabinets for the model plant Arabidopsis have been installed as part of the facility services.

One of the recently established Nordic phenomics facilities is the Finnish National Plant Phenotyping Infrastructure (NaPPI) founded in 2016. This facility is an example of a collaboration across two universities with a highthroughput phenomics facility at the University of Helsinki and a high-precision facility at the University of Eastern Finland (UEF) (https://www.helsinki.fi/en/infrastructures/national-plant-phenotyping/). The high-throughput facility consists of two units, one for small model plants like Arabidopsis and herbs, and one for larger crop plants, such as cereals, oil and protein crops, and small trees, up to $120 \mathrm{~cm}$ height. The small plants can be analyzed for growth, morphology and physiology by RGB cameras as well as thermal and PAM chlorophyll fluorescence (FluorCam) sensors. The large plant analysis unit consists of RGB cameras (top and 360 degree side view) and FluorCam. Both units allow automated plant management by weighing and watering, and transportation to imaging stations. 


\begin{tabular}{|c|c|c|c|c|c|c|c|c|c|c|c|c|c|c|}
\hline Name of facility & $\begin{array}{l}\text { PhenoDyn: } \\
\text { Drought } \\
\text { spotter and } \\
\text { Planteye }\end{array}$ & $\begin{array}{c}\text { Frederiksberg } \\
\text { facilities at } \\
\text { University of } \\
\text { Copenhagen }\end{array}$ & $\begin{array}{l}\text { Phenolab } \\
\text { Taasterup at } \\
\text { University of } \\
\text { Copenhagen }\end{array}$ & $\begin{array}{l}\text { Greenhouse } \\
\text { Taasterup, } \\
\text { University of } \\
\text { Copenhagen }\end{array}$ & $\begin{array}{l}\text { RERAF - Ris } \varnothing \\
\text { Environmental } \\
\text { Risk Assessment } \\
\text { facility }\end{array}$ & \begin{tabular}{|c|} 
Controlled \\
Environment \\
Facility for Plant \\
Research
\end{tabular} & $\begin{array}{l}\text { The Centre } \\
\text { for Plant } \\
\text { Research in } \\
\text { Controlled } \\
\text { Climate (SKP) }\end{array}$ & $\begin{array}{c}\text { Climate } \\
\text { laboratory Holt }\end{array}$ & $\begin{array}{l}\text { The biotron } \\
\text { at SLU Alnarp }\end{array}$ & $\begin{array}{l}\text { UPSC Growth } \\
\text { facility }\end{array}$ & $\begin{array}{l}\text { DEEP Plant } \\
\text { Growth } \\
\text { Facility }\end{array}$ & $\begin{array}{c}\text { Growing } \\
\text { facilities at } \\
\text { SLU Uppsala }\end{array}$ & $\begin{array}{c}\text { NaPPI small } \\
\text { plant unit, in } \\
\text { Viikki Plant } \\
\text { Growth } \\
\text { Facilities }\end{array}$ & $\begin{array}{c}\text { NaPPI large } \\
\text { plant unit, in } \\
\text { ViGoR facilities }\end{array}$ \\
\hline Host institution & $\begin{array}{l}\text { Food Science, } \\
\text { Aarhus } \\
\text { University }\end{array}$ & $\begin{array}{l}\text { PLEN, University } \\
\text { of Copenhagen }\end{array}$ & $\begin{array}{c}\text { PLEN, } \\
\text { University of } \\
\text { Copenhagen }\end{array}$ & $\begin{array}{l}\text { PLEN, University } \\
\text { of Copenhagen }\end{array}$ & $\begin{array}{l}\text { Inst for Env } \\
\text { engineering, } \\
\text { Technical } \\
\text { University of } \\
\text { Denmark }\end{array}$ & $\begin{array}{c}\text { Department } \\
\text { of Biosciences, } \\
\text { University of Oslo }\end{array}$ & $\begin{array}{l}\text { Norwegian } \\
\text { University of } \\
\text { Life Sciences } \\
\text { (NMBU) }\end{array}$ & $\begin{array}{l}\text { UiT The Arctic } \\
\text { University of } \\
\text { Norway }\end{array}$ & $\begin{array}{l}\text { LTV faculty, } \\
\text { SLU Alnarp }\end{array}$ & $\begin{array}{l}\text { Umeå Plant } \\
\text { Science } \\
\text { Centre }\end{array}$ & $\begin{array}{l}\text { Stockholm } \\
\text { University }\end{array}$ & $\begin{array}{l}\text { NJ faculty, } \\
\text { SLU Uppsala }\end{array}$ & $\begin{array}{c}\text { University } \\
\text { of Helsinki, } \\
\text { Finland }\end{array}$ & $\begin{array}{c}\text { University } \\
\text { of Helsinki, } \\
\text { Finland }\end{array}$ \\
\hline Type of facility & $\begin{array}{l}\text { Phenotyping } \\
\text { and controlled } \\
\text { environment }\end{array}$ & $\begin{array}{l}\text { Controlled } \\
\text { environment in } \\
\text { greenhouses }\end{array}$ & $\begin{array}{l}\text { Phenotyping } \\
\text { and controlled } \\
\text { environment }\end{array}$ & $\begin{array}{l}\text { Greenhouse with } \\
\text { controlled climate }\end{array}$ & $\begin{array}{l}\text { Controlled } \\
\text { environment }\end{array}$ & $\begin{array}{l}\text { Controlled } \\
\text { environment }\end{array}$ & $\begin{array}{c}\text { Controlled } \\
\text { environment, } \\
\text { test fields }\end{array}$ & \begin{tabular}{|c} 
Controlled \\
environment \\
And test fields
\end{tabular} & $\begin{array}{l}\text { Controlled } \\
\text { environment }\end{array}$ & $\begin{array}{l}\text { Phenotyping } \\
\text { and controlled } \\
\text { environment }\end{array}$ & $\begin{array}{l}\text { Phenotyping } \\
\text { and } \\
\text { controlled } \\
\text { environment }\end{array}$ & $\begin{array}{c}\text { Phenotyping } \\
\text { and } \\
\text { controlled } \\
\text { environment }\end{array}$ & $\begin{array}{c}\text { Small plant } \\
\text { phenotyping } \\
\text { controlled } \\
\text { environment }\end{array}$ & $\begin{array}{c}\text { Large plant } \\
\text { phenotyping } \\
\text { monitored } \\
\text { environment }\end{array}$ \\
\hline $\begin{array}{c}\text { Year } \\
\text { constructed }\end{array}$ & 2012-2015 & 1972-1984-1996 & 2015 & 2013 & $\begin{array}{c}\text { 1993, upgraded } \\
2003\end{array}$ & 1973 & 1995-2017 & 1978 & 2016 & $2002 / 2008$ & 2015/2016 & 2010-2012 & 2016 & 2016 \\
\hline $\begin{array}{c}\text { Type and } \\
\text { number of } \\
\text { chambers/ } \\
\text { units, size of } \\
\text { units }\end{array}$ & $\begin{array}{c}6 \text { climate } \\
\text { chambers, } \\
6 \text { full scale } \\
\text { greenhouse } \\
\text { cells }\end{array}$ & 15 chambers & $\begin{array}{c}117 \text { fixtures/ } \\
\text { plants }\end{array}$ & $\begin{array}{c}12 \text { compartments } \\
\left(50 \mathrm{~m}^{2}\right)\end{array}$ & $\begin{array}{c}6 \text { identical } \\
\text { chambers are } \\
\text { available (4 } \\
\left.{ }^{*} 6 * 3.1 \mathrm{~m}\right)\end{array}$ & $\begin{array}{c}16 \text { artifical } \\
\text { environments } \\
\left(10 \mathrm{~m}^{2}\right) \cdot 6 \\
\text { conditioned } \\
\text { natural daylight } \\
\left(\mathrm{CND} ; 30 \mathrm{~m}^{2}\right) \cdot 4 \\
\text { small chambers } \\
\left(1 \mathrm{~m}^{2}\right)\end{array}$ & $\begin{array}{c}22 \text { freezing } \\
\text { chambers } \\
\left(0.6-6.3 \mathrm{~m}^{2}\right) ; \\
15 \text { cooling } \\
\text { chambers } \\
(6.3- \\
\left.8.8 \mathrm{~m}^{2}\right) ; 62 \\
\text { greenhouse } \\
\text { rooms }(12- \\
\left.40 \mathrm{~m}^{2}\right) 16 \\
\text { phytotron } \\
\text { rooms }\left(12 \mathrm{~m}^{2}\right) ; \\
60 \text { growth } \\
\text { chambers } \\
\left(0.3-9 \mathrm{~m}^{2}\right)\end{array}$ & $\begin{array}{c}6 \text { day light } \\
\text { chamber } \\
\left(10,5 \mathrm{~m}^{2}\right) ; 3 \mathrm{x} \\
2 \text { dark rooms } \\
\left(3,6 \mathrm{~m}^{2}\right) ; 2 \mathrm{~s} 3 \\
\text { rooms, }\left(3,6 \mathrm{~m}^{2}\right) ; \\
3 \text { Cold rooms, } \\
\left(9,5 \mathrm{~m}^{2}\right)\end{array}$ & $\begin{array}{c}12 \text { Climatized } \\
\text { rooms }(\mathrm{CR} ; \\
\left.11.5 \mathrm{~m}^{2}\right) 4 \\
\text { Climatized } \\
\text { daylight } \\
\text { rooms }(\mathrm{DR} ; \\
\left.14 \mathrm{~m}^{2}\right) 4 \\
\text { Growth } \\
\text { rooms }(\mathrm{OR} ; \\
\left.8 \mathrm{~m}^{2}\right), 4 \\
\text { Greenhouse } \\
\text { rooms }(\mathrm{GR} ; \\
\left.14 \mathrm{~m}^{2}\right)\end{array}$ & $\begin{array}{l}8 \text { chambers } \\
\left(6 \mathrm{~m}^{2}\right) ; 18 \\
\text { growth rooms } \\
\left(10-13 \mathrm{~m}^{2}\right) ; 4 \\
\text { greenhouse } \\
\text { rooms }\left(24 \mathrm{~m}^{2}\right)\end{array}$ & $\begin{array}{c}4 \text { growth } \\
\text { chambers } \\
\left(5.7 \mathrm{~m}^{2}\right) \\
2 \text { Percival } \\
\text { growth } \\
\text { cabinets, } \\
1 \text { Binde in } \\
\text { vitro culture } \\
\text { growth } \\
\text { cabinet }\end{array}$ & $\begin{array}{l}12 \text { climate } \\
\text { chambers } \\
\left(5-1 \mathrm{~m}^{2}\right) ; \\
17 \text { growth } \\
\text { rooms; } 29 \\
\text { growth } \\
\text { cabinets. }\end{array}$ & $\begin{array}{l}1 \text { walk in } \\
\text { growth } \\
\text { chamber, } \\
27 \mathrm{~m}^{2} \\
1080 \text { plant } \\
\text { capacity }\end{array}$ & $\begin{array}{c}\text { One } 109 \mathrm{~m}^{2} \\
\text { greenhouse } \\
\text { space housing } \\
270 \text { plants in } \\
\text { 3-5 Liter pots }\end{array}$ \\
\hline $\begin{array}{c}\text { Type of light } \\
\text { sources } \\
\text { available and } \\
\text { range of a light } \\
\text { intensity ( } \mu \mathrm{mol} \\
\mathrm{m}^{-2} \mathrm{~s}^{-1} \text { ) }\end{array}$ & $\begin{array}{l}\text { LED near sun } \\
\text { up to } 900 \\
\text { in climate } \\
\text { chamber. } \\
\text { Ca } 300 \text { in } \\
\text { greenhouse } \\
\text { with SONT and } \\
\text { LED }\end{array}$ & $\begin{array}{l}\text { HPS (SON-T) } \\
\text { Minimum } 100\end{array}$ & $\begin{array}{c}\text { LED and } \\
\text { HPS (SON-T) } \\
\text { Minimum } 200\end{array}$ & $\begin{array}{l}\text { LED and HPS } \\
\text { (SON-T) Minimum } \\
200\end{array}$ & $\begin{array}{c}\text { Up to: } 400 \\
\text { New LED system } \\
\text { will be installed } \\
\text { in all chambers } \\
2018\end{array}$ & \begin{tabular}{|c|} 
Artificial \\
environment \\
$\max 300$ \\
Chamber max 400
\end{tabular} & $\begin{array}{c}\text { HPI, HQI, } \\
\text { SON-T, 50- } \\
\text { 200. LED } \\
\text { in some } \\
\text { chambers. } \\
\text { Natural } \\
\text { light in } \\
\text { phytotron and } \\
\text { greenhouse } \\
\text { room }\end{array}$ & \begin{tabular}{|c} 
LED lights \\
(0-3000, \\
fluorescent \\
lights max. 200 \\
natural light in \\
phytotrone
\end{tabular} & $\begin{array}{l}\text { CR: CDM } \\
\text { 75-600/LED } \\
\text { 50-600; DR: } \\
\text { assimilation } \\
\text { lighting } \\
\text { available; OR: } \\
\text { T5 fluorescent } \\
\text { tubes 50-250 }\end{array}$ & $\begin{array}{c}\text { High pressure } \\
\text { metal } \\
\text { halogen, } \max \\
\text { 750; LED in } \\
\text { greenhouse } \\
\text { rooms }\end{array}$ & $\begin{array}{l}\text { In growth } \\
\text { chamber is } \\
\text { from 0-500 }\end{array}$ & $\begin{array}{c}900 \text { in LED } \\
\text { cabinets } 300 \\
\text { in ventilated } \\
\text { boxes } 900\end{array}$ & $\begin{array}{c}\text { LED 0-500 } \\
\text { white light } \\
\text { with FarRed } \\
\text { option }\end{array}$ & $\begin{array}{l}\text { Natural light } \\
\text { (Plexiglas UV } \\
\text { transmitting), } \\
\text { high pressure } \\
\text { sodium lamps }\end{array}$ \\
\hline
\end{tabular}




\begin{tabular}{|c|c|c|c|c|c|c|c|c|c|c|c|c|c|c|}
\hline $\begin{array}{c}3 \mathrm{D} \\
\text { reconstruction }\end{array}$ & 3D cloud & No & No & No & No & No & No & No & No & no & No & No & $\begin{array}{l}\text { For individual } \\
\text { plants from two } \\
\text { side images }\end{array}$ & $\begin{array}{l}\text { Reconstructed } \\
\text { from top and } \\
\text { side views }\end{array}$ \\
\hline $\begin{array}{l}\text { Commonly } \\
\text { grown plants }\end{array}$ & $\begin{array}{l}\text { Wheat, tomato, } \\
\text { ornamentals }\end{array}$ & $\begin{array}{l}\text { Model and crop } \\
\text { plants; } \\
\text { Education- } \\
\text { Science- } \\
\text { Garden- } \\
\text { Summerflowers }\end{array}$ & $\begin{array}{l}\text { Model and } \\
\text { crop plants }\end{array}$ & $\begin{array}{l}\text { Model and crop } \\
\text { plants }\end{array}$ & $\begin{array}{l}\text { Varieties of barley, } \\
\text { oil seed rape and } \\
\text { wheat }\end{array}$ & $\begin{array}{l}\text { Arabidopsis, } \\
\text { Draba, Cochlearia, } \\
\text { Capsella, } \\
\text { Cardamina) Picea } \\
\text { spp. }\end{array}$ & $\begin{array}{l}\text { Model and } \\
\text { crop plants }\end{array}$ & $\begin{array}{l}\text { Root vegetables, } \\
\text { potato, berries, } \\
\text { grasses, tomato, } \\
\text { cuscuta, } \\
\text { Arabidosis, } \\
\text { woody species }\end{array}$ & $\begin{array}{c}\begin{array}{c}\text { Potato, } \\
\text { wheat, } \\
\text { Arabidopsis }\end{array} \\
\end{array}$ & $\begin{array}{l}\text { Arabidopsis, } \\
\text { hybrid aspen }\end{array}$ & $\begin{array}{l}\text { Arabidopsis, } \\
\text { poplar, } \\
\text { barley, } \\
\text { datisca }\end{array}$ & $\begin{array}{c}\text { Arabidopsis, } \\
\text { Capsella, } \\
\text { barley, } \\
\text { wheat, rape, } \\
\text { tomatoes, } \\
\text { tobacco, } \\
\text { rice, spruce, } \\
\text { pine }\end{array}$ & $\begin{array}{l}\text { Arabidopsis, } \\
\text { small herbs and } \\
\text { other plants, up } \\
\text { to } 40 \mathrm{~cm} \text { height }\end{array}$ & $\begin{array}{l}\text { cereals, crops, } \\
\text { small trees up to } \\
120 \mathrm{~cm} \text { height }\end{array}$ \\
\hline GMO certified & Yes & Yes & Yes & No & No & Yes & Yes & Yes & Yes & Yes & Yes & Yes & Yes & Yes \\
\hline $\begin{array}{l}\text { Plant } \\
\text { pathogens } \\
\text { allowed? }\end{array}$ & $\begin{array}{l}\text { Limited - } \\
\text { greenhouse ok }\end{array}$ & $\begin{array}{l}\text { Yes, upon } \\
\text { approval }\end{array}$ & $\begin{array}{l}\text { Yes, upon } \\
\text { approval }\end{array}$ & $\begin{array}{l}\text { Model and crop } \\
\text { plants }\end{array}$ & Yes, upon approval & Yes, upon approval & $\begin{array}{l}\text { Yes, upon } \\
\text { approval }\end{array}$ & $\begin{array}{l}\text { Yes, upon } \\
\text { approval }\end{array}$ & $\begin{array}{c}\text { Yes, upon } \\
\text { approval }\end{array}$ & No & $\begin{array}{c}\text { Some } \\
\text { pathogens }\end{array}$ & $\begin{array}{l}\text { Yes, upon } \\
\text { approval }\end{array}$ & $\begin{array}{l}\text { Yes, upon } \\
\text { approval }\end{array}$ & $\begin{array}{l}\text { Yes, upon } \\
\text { approval }\end{array}$ \\
\hline Accessibility & $\begin{array}{l}\text { Academic } \\
\text { researchers } \\
\text { outside } \\
\text { institution and } \\
\text { non-academia }\end{array}$ & $\begin{array}{l}\text { Academic } \\
\text { researchers } \\
\text { outside } \\
\text { institution and } \\
\text { non-academia }\end{array}$ & $\begin{array}{l}\text { Academic } \\
\text { researchers } \\
\text { outside } \\
\text { institution } \\
\text { and non- } \\
\text { academia }\end{array}$ & $\begin{array}{l}\text { Academic } \\
\text { researchers } \\
\text { outside } \\
\text { institution and } \\
\text { non-academia }\end{array}$ & $\begin{array}{l}\text { Academic } \\
\text { researchers outside } \\
\text { institution and } \\
\text { non-academia }\end{array}$ & $\begin{array}{c}\text { Academic } \\
\text { researchers } \\
\text { outside institution } \\
\text { and non-academia }\end{array}$ & $\begin{array}{c}\text { Academic } \\
\text { researchers } \\
\text { outside } \\
\text { institution, } \\
\text { non- } \\
\text { academia }\end{array}$ & $\begin{array}{l}\text { UiT and NIBIO } \\
\text { researchers; other } \\
\text { researchers via } \\
\text { collaboration }\end{array}$ & $\begin{array}{c}\text { Academic } \\
\text { researchers } \\
\text { outside } \\
\text { institution } \\
\text { and non- } \\
\text { academia }\end{array}$ & $\begin{array}{l}\text { Researchers } \\
\text { at UPSC } \\
\text { and if i i } \\
\text { collaboration }\end{array}$ & $\begin{array}{l}\text { Academic } \\
\text { researchers } \\
\text { only }\end{array}$ & $\begin{array}{l}\text { Academic } \\
\text { researchers } \\
\text { outside } \\
\text { institution } \\
\text { and non- } \\
\text { academia }\end{array}$ & $\begin{array}{l}\text { Academic } \\
\text { researchers } \\
\text { outside } \\
\text { institution and } \\
\text { non-academia }\end{array}$ & $\begin{array}{l}\text { Academic } \\
\text { researchers } \\
\text { outside } \\
\text { institution and } \\
\text { non-academia }\end{array}$ \\
\hline Contact details & $\begin{array}{l}\text { Carl-Otto } \\
\text { Ottosen, coo@ } \\
\text { food.au.dk }\end{array}$ & $\begin{array}{l}\text { www.plen. } \\
\text { ku.dk/om/pfv }\end{array}$ & $\begin{array}{c}\text { René Hvidberg } \\
\text { Petersen } \\
\text { rhvp@plen. } \\
\text { ku.dk } \\
\text { www.plen. } \\
\text { ku.dk/om/pfv }\end{array}$ & $\begin{array}{c}\text { René Hvidberg } \\
\text { Petersen rhvp@ } \\
\text { plen.ku.dk } \\
\text { www.plen.ku.dk/ } \\
\text { om/pfv }\end{array}$ & $\begin{array}{l}\text { Teis Nørgaard } \\
\text { Mikkelsen } \\
\text { temi@env.dtu.dk } \\
\text { www.env.dtu.dk }\end{array}$ & $\begin{array}{l}\text { http://www. } \\
\text { mn.uio.ono/ibv/ } \\
\text { english/research/ } \\
\text { sections/evogene/ } \\
\text { Infrastructure/ } \\
\text { phytotrone-plant- } \\
\text { facility/ }\end{array}$ & $\begin{array}{c}\frac{\mathrm{https} / / /}{\text { www. }} \\
\text { nmbu.no/ } \\
\text { tienester/ } \\
\text { sentre/skp }\end{array}$ & $\begin{array}{l}\text { https://en.uit. } \\
\text { no/forskning/ } \\
\text { forskningsgrupper/ } \\
\text { sub?p_pocument } \\
\text { id=341073\&sub_- } \\
\text { id=342049 } \\
\text { Laura Jakola } \\
\text { laura.jaakola@ } \\
\text { uit.no }\end{array}$ & $\begin{array}{c}\text { http:/// } \\
\text { www.slu. } \\
\text { se/en/ } \\
\text { biotronen/ }\end{array}$ & www.upsc.se & $\begin{array}{l}\text { Edouard. } \\
\text { pesquet@ } \\
\text { su.se }\end{array}$ & $\begin{array}{l}\text { Per Lindén } \\
\text { and Urban } \\
\text { Pettersson } \\
\text { Karin Eklund } \\
\text { and } \\
\text { Lars } \\
\text { Bergström }\end{array}$ & $\begin{array}{l}\text { Research } \\
\text { coordinator } \\
\text { Kristina } \\
\text { Himanen } \\
\text { https://www. } \\
\text { helsinkifif/en/ } \\
\text { infrastructures/ } \\
\text { national-plant- } \\
\text { phenotyping }\end{array}$ & $\begin{array}{c}\text { Research } \\
\text { coordinator } \\
\text { Kristina Himanen } \\
\text { https://www. } \\
\text { helsinkififen/ } \\
\text { infrastructures/ } \\
\text { national-plant- } \\
\text { phenotyping }\end{array}$ \\
\hline
\end{tabular}


These facilities allow analysis of 1080 small plants or 270 large plants simultaneously. The UEF NaPPI facility (Spectromics lab; http://www.uef.fi/web/spectromics) focuses on spectral imaging and development of advanced imaging technologies for plant phenotyping. The main instrumentation consists of four state-of-the-art hyperspectral imaging cameras covering an exceptionally wide range of electromagnetic radiation from ultraviolet to mid-wave infrared (250-5000 nm), three imaging PAMs for different scales (including microscopy), a range of illumination options, including a continuously tunable narrowband light source for macroscopic fluorescence, and sensitive cameras optimized for specific applications, such as deep UV imaging.

As an example of new, advanced climate chambers, the recently inaugurated Biotron at SLU Alnarp (http://www. slu.se/en/faculties/ltv/resurser1/biotron/) offers a very precise climate and light conditions also for large plants and with some chambers equipped with wavelength adjustable LED lights, even if it lacks high-throughput phenotyping. The Biotron has 28 chambers grouped into four different types based on their features. The temperature range in the chambers can be adjusted from $-5{ }^{\circ} \mathrm{C}$ to $+45^{\circ} \mathrm{C}$. The light intensity can be adjusted in the range of $50-600 \mu \mathrm{mol} \mathrm{m}^{-2} \mathrm{~s}^{-1}$ and the chambers with the LED lights can be altered for different spectral signatures. The air humidity and $\mathrm{CO}_{2}$ levels can also be altered.

Today, new LED sources allow simulation of outdoor light in controlled environments and several of the Nordic facilities now provide this option also with adjustable wavelength composition (Table 1). Such facilities allow application of the specific Nordic long photoperiods with reduced red to far-red ratios in light quality (Jaakola and Hohtola 2010). Advanced phenotyping in such controlled environments will be important for applications in greenhouse farming, which has high relevance in plant production in the Nordic countries. There are also several plants economically important for the Nordic countries which are difficult to study in controlled climates because of their perenniality, size or below-ground production. For example, it is known that in potato tubers certain phenylpropanoids and carotenoids are effected by both light quality and day length, and consequently differ depending growth latitude (Payyavula et al. 2012). In addition to the facilities listed in Table 1, there are several specialized facilities such as the RadiMax at University of Copenhagen and Weiss freezing unit at NMBU, which can operate with high precision between -80 to $+60^{\circ} \mathrm{C}$. The unique RadiMax field facility contains 600 so called minirhizotrons, which allows for semi-automated observation of roots from 0.5 to $3 \mathrm{~m}$ depth. The root imaging can be combined with above-ground drone imaging and watering is controlled with a root irrigation system and rainout shelters.

\section{Field phenotyping to excel Nordic research}

It is well-known that developmental and morphological differences caused by the artificial environment imposed in a laboratory setting can mask important crop traits. For example, a recent meta-analysis by Poorter et al. (2016) found that greenhouse-grown plants grew faster, had higher nitrogen concentrations and a different morphology than when grown in the field. Also light in field conditions has a large impact on plant growth. For example, the model plant Arabidopsis displays changed leaf morphology with altered pigment composition and fitness performance when grown outdoors (Mishra et al. 2012). These findings highlight the importance of replicating experiments in field conditions.

Still, the dynamic nature of the environment remains one of the largest hurdles in field phenotyping, and in Nordic region weather fluctuations are extreme both within a year and between years (Peltonen-Sainio 2012). Field experiments are also linked to challenges in representative sampling of plant material with larger variation, practical logistics issues working outside the standard lab infrastructure, and measuring the multi-stress environment in the field, and taking these into account when analyzing the data (Alexandersson et al. 2014, George et al. 2014). There is a tendency to oversimplify the contribution of the environment, especially when it comes to soil conditions, on the phenotype. To this end, so called envirotyping providing detailed information on many environmental parameters has been brought forward (Pauli et al. 2016). Even if the outdoor multi-stress condition is one of the major challenges, field experiments still offer the possibility to study the effects of a dynamic, multi-stress environments impossible to set up in the lab. It is known that a combination of stresses can lead to responses on the molecular level distinct from single environmental cues (Suzuki et al. 2014). Furthermore, in plant protection biology, field resistance is a common term for resistance that is not detectable in the lab and only appears under field conditions. Since field resistance can be assumed to be climate-dependent it needs to be studied in the specific climates such as in Nordic conditions. With more standardized systems for field phenotyping and improved climate facilities the mechanisms behind it can be studied more accurately. Improved field phenomics will also be important for precision farming and remote sensing for detection of nutrient deficiency and pathogen attacks. 
There are several efforts ongoing to improve field phenotyping efforts in the Nordic countries including manipulations in field. Norwegian University of Life Sciences (NMBU) is for example working to upgrade the field phenotyping facilities at Vollebekk. Field phenotyping technologies are currently being developed in-house based on the NMBU-developed Thorvald robotic platform and Unmanned aerial vehicles (UAVs) fitted with multispectral and hyperspectral cameras. A mist irrigation system is available for controlling humidity in the field and future plans include construction of rain shelters for further manipulation of field trial environments (https://www.nmbu.no/ tjenester/sentre/skp). In Sweden, the nationally co-ordinated infrastructure SITES (www.fieldsites.se) is currently investing in new drones and imaging equipment for their field stations. Also several cross-disciplinary projects are currently ongoing in the Nordic countries, for example EnBlightMe (https://www.vinnova.se/p/enblightme--ett-automatiserat-stodsystem-for-upptackt-av-potatisbladmogel/), which strives to detect late blight disease in potato crops, involves both plant pathologists and computer programmers.

Early on it was highlighted that a range of questions can be tackled in ecological, biodiversity, and climate change research using remote sensing (Kerr and Ostrovsky 2003). UAVs generally form an important part of the field phenotyping toolbox by equipping them with imaging devices (Fahlgren et al. 2015, Großkinsky et al. 2015). There are several Nordic research institutions and companies which are in the forefront of both UAV technique, image analysis and solutions for precision agriculture, areas which can all be connected to plant field phenotyping. Commercial drones can be mounted with various cameras for RGB, NDVI, multispectral or thermal imaging that can be used for evaluating canopy surface temperature, photosystem II activity, stomatal conductance and other physiological characteristics. In the field studies, remote sensing with UAVs and satellite images are becoming increasingly popular for use in agriculture due to reduced costs of obtaining the data and availability of free software for the analysis. The RGB cameras are the most affordable option albeit hold some limitations compared to other sensors. The images from drones can be processed in the open source software opendronemap (www.opendronemap.org) or other commercial software. Using satellite images from Denmark, Norway and Sweden, CropSAT (www.cropsat.se) quantifies the variation in the biomass for a given area. This data can be freely downloaded from the website to the on-farm equipment for variable spraying of fertilizer in the field.

\section{The low-cost phenotyping tools allow extending the phenotyping experiments}

Infrastructures for high-throughput sensor-based phenotyping have proven to be extremely useful for screening plants for traits of interest. However, due to the high costs associated with establishing such a system compounded with high maintenance costs, low-cost semi-automatic systems could be a valuable alternative, also for many Nordic institutions. Tailored, mobile low-cost solutions could be especially helpful and good solutions for Nordic academic institutions where long distances between facilities hampers the setting up of experiments at the few larger facilities available.

A low-cost imaging system can be modular, which allows inclusion of new cameras and sensors incrementally. An entry-level system can include an evenly lit imaging platform with a white, blue or black background lit with two studio strobes one on each side and two entry-level digital cameras for side and top view imaging of plants. Optionally, NDVI, near-infrared, microbolometer-based thermal cameras or chlorophyll fluorescence cameras can be attached to the system. As an example, a low-cost phenotyping lab (LCP Lab) with two RGB cameras and plant tracking with QR code has been setup at the Department of Plant Breeding at SLU with a capacity to photograph up-to a few hundred plants a day. At the University of Eastern Finland, an automated low-cost platform (SmartLab Plants) for monitoring plants with RGB, thermal, and motorized filter-based NDVI camera has been set up with Arduino-based controllers. Several open source or freeware software are available to analyze the images for measuring various morphological traits. Examples of software that can be installed on local computers are HTPheno (Hartmann et al. 2011), Integrated Analysis Platform (IAP) (Klukas et al. 2014), ImageHarvest (Knecht et al. 2016), Plant Computer Vision (PlantCV) (Fahlgren et al. 2015) and Easy Leaf Area (Easlon and Bloom 2014). These software vary in their user friendliness, available features, computational resource requirements and the traits measured. Using such a system, traits such as early vigor, plant height and width, and growth rate can be measured. Thus, low-cost phenotyping both in the controlled conditions and in the field is a promising resource for effective analysis of agronomic traits in crops when sensor calibration, environmental data integration, and data standardization is organized.

\section{Educational and networking efforts for the Nordic countries}

In the Nordic countries, science and innovation capacity should be mobilized to meet the needs of a more climate resilient agriculture and forestry sector. We need to develop modern breeding approaches that utilize the expanding genomic knowledge on the one hand and the application of the new phenomics facilities on the other. 
Phenomics is rapidly developing at the moment both on the technological and research side with recently established facilities for both phenotyping and climate control. The main recent developments are better imaging methods, cheaper and more efficient acquisition of large-scale data sets and improved modelling capacity. Still, better interdisciplinary efforts and achievements addressing challenges specific for the Nordic countries are needed, as climate change imposes specific challenges to Nordic climates.

The recent establishment of plant facilities has opened new possibilities for collaboration between the Nordic countries but also internationally. The International Plant Phenotyping Network (IPPN) offers specialist working groups and international networking between global facilities (https://www.plant-phenotyping.org/), European ESFRI project EMPHASIS aims at building a pan-European plant phenotyping infrastructure with shared standards (https://emphasis.plant-phenotyping.eu/), the Horizon2020 project EPPN2020 provides transnational access to the European plant phenotyping facilities (https://eppn2020.plant-phenotyping.eu/), something Nordic researchers should benefit from. In the Nordic region, a university hub called NordPlant was recently established for shared education, research and mobility efforts associated with local plant research facilities (www.nordplant. org). Another Nordic network, the Nordic Plant Phenotyping Network (NPPN; www. nordicphenotyping.org) is active in developing lean technologies directed for the end users, breeders and farmers. Recently, NOVA University network granted funding for a 4 year PhD course series (2018-2021) called "Phenotyping Technologies in Plantenvironment Interactions", which forms an important joint educational platform for the Nordic plant research community (https://www.nmbu.no/en/students/nova/students/phd-courses/phd-courses-2018/node/31463).

We hope that these networks encourage Nordic plant researchers to strengthen their research efforts through:

- $\quad$ sharing research infrastructures and improving interoperability

- promoting researcher mobility

- $\quad$ providing controlled climate and phenotyping platforms for groups of plant researchers currently not using them regularly, such as plant ecologists and botanists

- $\quad$ establishing a forum for developing low-cost phenotyping facilities

- $\quad$ synchronizing educational efforts

- evolving and unifying technological development

- drive innovation together with private enterprises

- opening Nordic crop repositories and sharing Nordic data

- $\quad$ aligning activities with European and global initiatives and networks

\section{Acknowledgements}

This work was supported by funding from Vinnova to EA (2016-04386), from Juho and Lempi Pitkänen Fund to MK, from HiLIFE and EU H2020 project No 731013 (EPPN2020) to KH, from Lantmännen Research Foundation and Partnerskap Alnarp to AC, and NordPlant (NordForsk: 84597) to EA, AC and KH. We thank Morten Lillemo and other facility managers and co-workers (Table 1) for contributing with information about their infrastructures.

\section{References}

Alexandersson, E., Jacobson, D., Vivier, M.A., Weckwerth, W. \& Andreasson, E. 2014. Field-omics-understanding large-scale molecular data from field crops. Frontiers in Plant Science 5: 286. https://doi.org/10.3389/fpls.2014.00286

Andreasen, C. \& Streibig, J.C. 2011. Evaluation of changes in weed flora in arable fields of Nordic countries-based on Danish longterm surveys. Weed Research 51: 214-226. https://doi.org/10.1111/j.1365-3180.2010.00836.x

Arend, D., Junker, A., Scholz, U., Schule, D., Wylie, J. \& Lange, M. 2016. PGP repository: a plant phenomics and genomics data publications infrastucture. Database, Volume 2016, 1 January 2016, baw033. https://DOI: 10.1093/database/baw033

Bjerke, J.W., Karlsen, S.R., Høgda, K.A., Malnes, E., Jepsen, J.U., Lovibond, S., Vikhamar-Schuler, D. \& Tømmervik, H. 2014. Recordlow primary productivity and high plant damage in the Nordic Arctic Region in 2012 caused by multiple weather events and pest outbreaks. Environmental Research Letters 9: 084006. https://doi.org/10.1088/1748-9326/9/8/084006

Coppens, F., Wuyts, N., Inze, D. \& Dhondt, S. 2017. Unlocking the potential of plant phenotyping data through integration and data-driven approaches. Current Opinion in Systems Biology 4: 58-63. https://doi.org/10.1016/j.coisb.2017.07.002

Deepak, M., Lihavainen, J., Keski-Saari, S., Kontunen-Soppela, S., Salojärvi, J., Tenkanen, A., Heimonen, K., Oksanen, E. \& Keinänen, M. 2018. Genotype and provenance-related variation in the leaf surface secondary metabolites of silver birch. Canadian Journal of Forest Research 48: 1-11. https://doi.org/10.1139/cjfr-2017-0446

Dumont, J., Hirvonen, T., Heikkinen, V., Mistretta, M., Granlund, L., Himanen, K., Fauch, L., Porali, I., Hiltunen, J., Keski-Saari, S., Nygren, M., Oksanen, E., Hauta-Kasari, M. \& Keinänen, M. 2015. Thermal and hyperspectral imaging for Norway spruce (Picea abies) seeds screening. Computers and Electronics in Agriculture 116: 118-124.https://doi.org/10.1016/j.compag.2015.06.010 
E. Alexandersson et al. (2018) 27: 7-16

Easlon, H.M. \& Bloom, A.J. 2014. Easy Leaf Area: Automated Digital Image Analysis for Rapid and Accurate Measurement of Leaf Area. Applications in Plant Sciences 2: 1400033. https://doi.org/10.3732/apps.1400033

Fahlgren, N., Gehan, M.A. \& Baxter, I. 2015. Lights, camera, action: high-throughput plant phenotyping is ready for a close-up. Current Opinion in Plant Biology 24: 93-99. https://doi.org/10.1016/j.pbi.2015.02.006

George, T.S., Hawes, C., Newton, A.C., McKenzie, B.M., Hallett, P.D. \& Valentine, T.A. 2014. Field phenotyping and long-term platforms to characterise how crop genotypes interact with soil processes and the environment. Agronomy 4: 242-278. https://doi. org/10.3390/agronomy4020242

Großkinsky, D.K., Svensgaard, J., Christensen, S. \& Roitsch, T. 2015. Plant phenomics and the need for physiological phenotyping across scales to narrow the genotype-to-phenotype knowledge gap. Journal of Experimental Botany 66: 5429-5440. https://doi. org/10.1093/jxb/erv345

Hartmann, A., Czauderna, T., Hoffmann, R., Stein, N. \& Schreiber, F. 2011. HTPheno: An image analysis pipeline for high-throughput plant phenotyping. BMC Bioinformatics 12: 148. https://doi.org/10.1186/1471-2105-12-148

Humplík, J.F., Lazár, D., Fürst, T., Husičková, A., Hýbl, M. \& Spíchal, L. 2015. Automated integrative high-throughput phenotyping of plant shoots: a case study of the cold-tolerance of pea (Pisum sativum L.). Plant Methods 11: 20. https://doi.org/10.1186/ s13007-015-0063-9

IPCC 2013. Climate change 2013: the physical science basis. In: Stocker, T.F., Qin, D., Plattner, G.-K., Tignor, M., Allen, S.K., Boschung, J., Nauels, A., Xia, Y. Bex V. \& Midgley, P.M. (eds.). Contribution of Working Group I to the Fifth Assessment Report of the Intergovernmental Panel on Climate Change. Cambridge, United Kingdom and New York, NY, USA: Cambridge University Press. 1535 p.

Jaakola, L. \& Hohtola, A. 2010. Effect of latitude on flavonoid biosynthesis in plants. Plant, Cell \& Environment 33: 1239-1247. https://doi.org/10.1111/j.1365-3040.2010.02154.x

Kerr, J.T. \& Ostrovsky, M. 2003. From space to species: ecological applications for remote sensing. Trends in Ecology \& Evolution 18: 299-305. https://doi.org/10.1016/S0169-5347(03)00071-5

Klukas, C., Chen, D. \& Pape, J.M. 2014. Integrated Analysis Platform: An Open-Source Information System for High-Throughput Plant Phenotyping. Plant Physiology 165: 506-518. https://doi.org/10.1104/pp.113.233932

Knecht, A.C., Campbell, M.T., Caprez, A., Swanson, D.R. \& Walia, H. 2016. Image Harvest: an open-source platform for high-throughput plant image processing and analysis. Journal of Experimental Botany 67: 3587-3599. https://doi.org/10.1093/jxb/erw176

Krajewski, P., Chen, D., Ćwiek, H., van Dijk, A.D.J., Fiorani, F., Kersey, P., Klukas, C., Lange, M., Markiewicz, A., Nap, J.P., van Oeveren, J., Pommier, C., Scholz, U., van Schriek, M., Usadel, B. \& Weise, S. 2015. Towards recommendations for metadata and data handling in plant phenotyping. Journal of Experimental Botany 66: 5417-5427.https://doi.org/10.1093/jxb/erv271

Lankinen, A.., Abreha, K.B., Alexandersson, E., Andersson, S. \& Andreasson, E. 2016. Nongenetic Inheritance of Induced Resistance in a Wild Annual Plant. Phytopathology 106: 877-883. https://doi.org/10.1094/PHYTO-10-15-0278-R

Laughlin, D.C. \& Messier, J. 2015. Fitness of multidimensional phenotypes in dynamic adaptive landscapes. Trends in Ecology \& Evolution 30: 487-496. https://doi.org/10.1016/j.tree.2015.06.003

Li, Y., Huang, Y., Bergelson, J., Nordborg, M. \& Borevitz, J.O. 2010. Association mapping of local climate-sensitive quantitative trait loci in Arabidopsis thaliana. Proceedings of the National Academy of Sciences 107: 21199-21204.https://doi.org/10.1073/ pnas. 1007431107

Lihavainen, J., Ahonen, V., Keski-Saari, S., Sõber, A., Oksanen, E. \& Keinänen, M. 2017. Low vapor pressure deficit reduces glandular trichome density and modifies the chemical composition of cuticular waxes in silver birch leaves. Tree Physiology 37:11661181. https://doi.org/10.1093/treephys/tpx045

Mishra, Y., Johansson Jänkänpää, H., Kiss, A.Z., Funk, C., Schröder, W.P. \& Jansson, S. 2012. Arabidopsis plants grown in the field and climate chambers significantly differ in leaf morphology and photosystem components. BMC Plant Biology 12: 6. https://doi. org/10.1186/1471-2229-12-6

Pauli, D., Andrade-Sanchez, P., Carmo-Silva, A.E., Gazave, E., French, A.N., Heun, J., Hunsaker, D.J., Lipka, A.E., Setter, T.L., Strand, R.J., Thorp, K.R., Wang, S., White, J.W. \& Gore, M.A. 2016. Field-Based High-Throughput Plant Phenotyping Reveals the Temporal Patterns of Quantitative Trait Loci Associated with Stress-Responsive Traits in Cotton. G3 Genes/Genomes/Genetics 6: 865-879. https://doi.org/10.1534/g3.115.023515

Pavicic, M., Mouhu, K., Wang, F., Bilicka, M., Chovancek, E. \& Himanen, K. 2017. Genomic and Phenomic Screens for Flower Related RING Type Ubiquitin E3 Ligases in Arabidopsis. Frontiers in Plant Science 8: 416. https://doi.org/10.3389/fpls.2017.00416

Payyavula, R., Navarre, D., Kuhl, J., Pantoja, A. \& Pillai, S. 2012. Differential effects of environment on potato phenylpropanoid and carotenoid expression. BMC Plant Biology 12: 39. https://doi.org/10.1186/1471-2229-12-39

Peltonen-Sainio, P. 2012. Crop production in a northern climate. In: Meybeck, A., Lankoski, J., Redfern, S., Azzu, N. \& Gitz, V. (eds.). Building resilience for adaptation to climate change in the agriculture sector. Proceedings of a joint FAO/OECD workshop April 2012. FAO 2012, Rome. ISBN 978-92-5-107373-5

Poorter, L., Bongers, F., Aide, T.M., Almeyda Zambrano, A.M., Balvanera, P., Becknell, J.M., Boukili, V., Brancalion, P.H., Broadbent, E.N., Chazdon, R.L., Craven, D., de Almeida-Cortez, J.S., Cabral, G.A., de Jong, B.H., Denslow, J.S., Dent, D.H., DeWalt, S.J., Dupuy, J.M., Duran, S.M., Espirito-Santo, M.M., Fandino, M.C., Cesar, R.G., Hall, J.S., Hernandez-Stefanoni, J.L., Jakovac, C.C., Junqueira, A.B., Kennard, D., Letcher, S.G., Licona, J.C., Lohbeck, M., Marin-Spiotta, E., Martinez-Ramos, M., Massoca, P., Meave, J.A., Mesquita, R., Mora, F., Munoz, R., Muscarella, R., Nunes, Y.R., Ochoa-Gaona, S., de Oliveira, A.A., Orihuela-Belmonte, E., Pena-Claros, M., Perez-Garcia, E.A., Piotto, D., Powers, J.S., Rodriguez-Velazquez, J., Romero-Perez, I.E., Ruiz, J., Saldarriaga, J.G., Sanchez-Azofeifa, A., Schwartz, N.B., Steininger, M.K., Swenson, N.G., Toledo, M., Uriarte, M., van Breugel, M., van der Wal, H., Veloso, M.D., Vester, H.F., Vicentini, A., Vieira, I.C., Bentos, T.V., Williamson, G.B. \& Rozendaal, D.M. 2016. Biomass resilience of Neotropical secondary forests. Nature 530: 211-214. https://doi.org/10.1038/nature16512

Roos, J., Hopkins, R., Kvarnheden, A. \& Dixelius, C. 2011. The impact of global warming on plant diseases and insect vectors in Sweden. European Journal of Plant Pathology 129: 9-19. https://doi.org/10.1007/s10658-010-9692-z 
Sellin, A., Alber, M., Keinänen, M., Kupper, P., Lihavainen, J., Lõhmus, K., Oksanen, E., Sõber, A., Sõber, J. \& Tullus, A. 2017. Growth of northern deciduous trees under increasing atmospheric humidity: possible mechanisms behind the growth retardation. Regional Environmental Change 17: 2135-2148. https://doi.org/10.1007/s10113-016-1042-z

Shipley, B., De Bello, F., Cornelissen, J.H.C., Laliberté, E., Laughlin, D.C. \& Reich, P.B. 2016. Reinforcing loose foundation stones in trait-based plant ecology. Oecologia 180: 923-931. https://doi.org/10.1007/s00442-016-3549-x

Stanford, N.J., Wolstencroft, K., Golebiewski, M., Kania, R., Juty, N., Tomlinson, C., Owen, S., Butcher, S., Hermjakob, H., Le Novere, N., Mueller, W., Snoep, J. \& Goble, C. 2015. The evolution of standards and data management practices in systems biology. Molecular Systems Biology 11: 851. https://doi.org/10.15252/msb.20156053

Suzuki, N., Rivero, R.M., Shulaev, V., Blumwald, E. \& Mittler, R. 2014. Abiotic and biotic stress combinations. New Phytologist 203: 32-43. https://doi.org/10.1111/nph.12797

van der Fels-Klerx, H., van Asselt, E.D., Madsen, M.S. \& Olesen, J.E. 2013. Impact of climate change effects on contamination of cereal grains with deoxynivalenol. PloS One 8: e73602. https://doi.org/10.1371/journal.pone.0073602

Vasseur, F., Wang, G., Bresson, J., Schwab, R. \& Weigel, D. 2017. Image-based methods for phenotyping growth dynamics and fitness in large plant populations. bioRxiv. https://doi.org/10.1101/208512

Wahabzada, M., Mahlein, A.-K., Bauckhage, C., Steiner, U., Oerke, E.C. \& Kersting, K. 2015. Metro Maps of Plant Disease Dynamics-Automated Mining of Differences Using Hyperspectral Images. Plos One 10: e0116902. https://doi.org/10.1371/journal. pone.0116902

Wheeler, T. \& von Braun, J. 2013. Climate Change Impacts on Global Food Security. Science 341: 508-513. https://doi.org/10.1126/ science.1239402 